\title{
Ionic conductivity in Sm-doped ceria from first- principles non-equilibrium molecular dynamics
}

Johan Klarbring, Olga Yu. Vekilova, Johan O. Nilsson, Natalia V. Skorodumova and Sergey Simak

\author{
Journal Article
}

\section{Tweet}

N.B.: When citing this work, cite the original article.

Original Publication:

Johan Klarbring, Olga Yu. Vekilova, Johan O. Nilsson, Natalia V. Skorodumova and Sergey Simak, Ionic conductivity in Sm-doped ceria from first-principles non-equilibrium molecular dynamics, Solid State Ionics, 2016. 296, pp.47-53.

http://dx.doi.org/10.1016/j.ssi.2016.08.011

Copyright: Elsevier

http://www.elsevier.com/

Postprint available at: Linköping University Electronic Press

http://urn.kb.se/resolve?urn=urn:nbn:se:liu:diva-132831

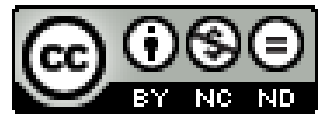




\title{
Ionic conductivity in Sm-doped ceria from first-principles non-equilibrium molecular dynamics
}

\author{
Johan Klarbring, ${ }^{1, *}$ Olga Yu. Vekilova, ${ }^{2,3}$ Johan O. Nilsson, ${ }^{2}$ Natalia V. Skorodumova,,${ }^{2,3}$ and Sergei I. Simak ${ }^{1}$ \\ ${ }^{1}$ Department of Physics, Chemistry and Biology (IFM), \\ Linköping University, SE-581 83, Linköping, Sweden \\ ${ }^{2}$ Materials Science and Engineering Department, \\ KTH-Royal Institute of Technology, Brinellvägen 13, 10044 Stockholm, Sweden \\ ${ }^{3}$ Department of Physics and Astronomy, Uppsala University, Box 516, 75120 Uppsala, Sweden
}

\begin{abstract}
$\mathrm{Sm}$-doped ceria is a prospective electrolyte material for intermediate-temperature solid-oxide fuel cells (IT-SOFC). Equilibrium ab initio molecular dynamics (AIMD) studies of oxygen ion diffusion in this material are currently impractical due to the rareness of diffusive events on the accessible timescale. To overcome this issue we have performed $a b$ initio non-equilibrium molecular dynamics calculations of Sm-doped ceria using the color-diffusion algorithm. Applying an external force field we have been able to increase the frequency of diffusive events over the simulation time, while keeping the physical mechanism of diffusion intact. We have investigated the temperature dependence of the maximum strength of the applied external field that could be used while maintaining the response of the system in a linear regime. This allows one to obtain the diffusivity at zero field. The bulk ionic conductivity has been calculated and found to match the experimental data well. We have also compared the description of the diffusion process by our method to previous findings and show that the migration mechanism and site preference of oxygen vacancies with respect to the Sm dopants is well reproduced.
\end{abstract}

\section{INTRODUCTION}

Ceria, $\mathrm{CeO}_{2}$, has been a subject of comprehensive study for the last few decades, largely due to a variety of intriguing properties, allowing its use in areas such as catalysis $^{1}$ and fuel cell technology ${ }^{2}$. When doped with rare earth (RE) cations of lower valence, oxygen vacancies are introduced in the anion sublattice resulting in high mobility of oxygen ions, leading to high ionic conductivity. This makes RE-doped ceria a promising candidate as an electrolyte material in intermediate temperature $\left(500-700^{\circ} \mathrm{C}\right)$ solid oxide fuel cells $(\mathrm{SOFC})^{2}$. In particular Sm-doped ceria (SDC) has been shown to exhibit one of the highest conductivities of all ceria based materials ${ }^{3,4}$.

A vast amount of both experimental ${ }^{3-6}$ and computational $^{7-21}$ studies have been performed attempting to describe and find ways to enhance the ionic conductivity of ceria based materials. Previous computational studies include classical molecular dynamics $(\mathrm{MD})^{7,11-13}$ and kinetic Monte Carlo $(\mathrm{KMC})^{8,10,16}$ simulations, as well as first-principles calculations based on density functional theory (DFT) $)^{9,14,18,22}$.

Classical MD simulations rely on empirical potential models for the interactions of the atoms in the studied system. They have the advantage of explicitly treating temperature effects at a moderate computational cost, allowing for long simulation times for a large number of atoms. This approach, however, suffers drawbacks in transferability and the quality of the calculations is totally dependent on how well the assumed potential model describes the actual system. In particular, model potentials commonly fail when used to describe properties outside the realm for which they were parametrized; an example for ceria is found in Ref. 17.
In the first-principles, or ab initio, approach one starts directly from the laws of quantum mechanics, resulting in a more accurate and universal description of the interactions in the system. Unfortunately, the expensive nature of $a b$ initio MD (AIMD) simulations, where the atomic interactions are calculated using, for example, DFT, makes the simulation times required to properly capture the diffusion process under equilibrium conditions to long to be practical. This is due to the mismatch of timescales between that of the atomic vibrations and that of the much longer process of ionic diffusion.

DFT studies on doped ceria have therefore largely been focused on calculating energy barriers for oxygen migration at $T=0 \mathrm{~K}$. These can then be inserted into an Arrhenius expression for ionic conductivity, or be taken as an input into the kinetic Monte Carlo (KMC) scheme where they are used to calculate transition rates between configurations of the system separated by an oxide ion migration event. Although this method allows one to collect good statistics and model large systems it relies on the assumption that the potential surface determined for $0 \mathrm{~K}$ is not altered by high temperature.

In this work we address this issue using the nonequilibrium MD (NEMD) approach, more specifically the color-diffusion algorithm ${ }^{23}$, where the diffusion process is accelerated by applying an external force field, which makes it possible to properly capture it on timescales accessible to AIMD. The color-diffusion algorithm was originally used in combination with model potentials to study liquids $^{23}$, and was only recently merged with AIMD to study diffusion of $\mathrm{Li}$ ions in solid $\mathrm{LiBH}_{4}$, by Aeberhard et al. ${ }^{24}$, and later by us to study the ionic conductivity in Gd-doped ceria ${ }^{25}$.

We have applied our version of the color-diffusion algorithm ${ }^{25}$ to study the diffusion of oxygen ions in Sm- 
doped ceria from first principles. We have calculated the temperature dependence of the ionic conductivity and further investigated how well the diffusion process is reproduced by our method by investigating the microscopic details, such as migration path and vacancy site preference, of the oxygen ion diffusion.

\section{METHODOLOGY}

\section{A. The Color-Diffusion Algorithm}

Our NEMD calculations are based on the colordiffusion algorithm ${ }^{23}$ where one applies an external "color" field, $\mathbf{F}_{e}$ to the system under study, and assigns each atom, $i=1, \ldots, N$, a color charge, $c_{i}$. The extra force felt by atom $i$ due to the external field is $c_{i} \mathbf{F}_{e}$. The non-diffusive atoms are given color charge $c_{i}=0$ and are therefore not directly influenced by the external field. The diffusive atoms are, for example ${ }^{24}$, given color charge $c_{i}=1$ or $c_{i}=-1$, so they feel a force in the same or opposite direction as the external field, respectively. The distribution of color charges among the diffusive atoms, as well as the direction and strength of $\mathbf{F}_{e}$, are system specific.

The external field alters the standard AIMD equations of motion (EOM) to look as follows

$$
\begin{aligned}
\dot{\mathbf{q}}_{i} & =\frac{\mathbf{p}_{i}}{m_{i}}, \\
\dot{\mathbf{p}}_{i} & =\mathbf{F}_{i}^{D F T}+c_{i} \mathbf{F}_{e}-\frac{m_{i}}{M} \mathbf{F}_{t o t},
\end{aligned}
$$

where $\mathbf{q}_{i}$ and $\mathbf{p}_{i}$ are the position and momentum of atom $i$, respectively, $\mathbf{F}_{i}^{D F T}$ is the force obtained from DFT in a standard AIMD simulation. $\mathbf{F}_{\text {tot }}=\sum c_{i} \mathbf{F}_{e}$ is the total force on the system and the inclusion of the term $\frac{m_{i}}{M} \mathbf{F}_{t o t}$, where $m_{i}$ is the mass of atom $i$ and $M$ is the system's total mass, thus removes any net force on the system, fixing the reference frame. Note that this term vanishes in the case of color charge neutrality $\left(\sum c_{i}=0\right)$ and is therefore only needed in the case of a non-zero total color charge $\left(\sum c_{i} \neq 0\right)$.

In Sm-doped $\mathrm{CeO}_{2}$ diffusion of the cations is very limited; we therefore give the Ce and Sm atoms color charge zero. Oxygen diffusion in ceria and doped ceria is known to occur by a vacancy hopping mechanism along all $\langle 100\rangle$ directions of the anion sublattice. The direction of the external field is therefore set in the [111] direction to avoid promoting the diffusion more in any one of the $\langle 100\rangle$ directions than the others. All oxygen atoms are given $c_{i}=1 .^{25}$

Continuous application of the external field will, if the temperature is not controlled, indefinitely heat up the system. This can be avoided by applying a thermostat. We employ a velocity scaling (VS) thermostat. Typically, the VS thermostat scales the velocities of all the atoms in the system to match a desired temperature at each time step. In our methodology, to avoid possible direct counteracting effects between the external field and the thermostat, only the velocities of the Ce and Sm atoms are scaled, and the temperature of the oxygen subsystem is controlled indirectly by its heat exchange with the $\mathrm{Ce} / \mathrm{Sm}$ subsystem. Such VS results in an average temperature which is somewhat $(\sim 50 \mathrm{~K})$ different from the starting temperature. We attribute this difference to the mechanism of the thermostat. Since the VS thermostat is only applied to the Ce/Sm subsystem, the actual average temperature of the simulations (of all the atoms) slightly differs from the initial temperature. Only scaling the $\mathrm{Ce} / \mathrm{Sm}$ velocities will also in general lead to a change of the system's total momentum ${ }^{26}$. To fix the total momentum at zero, the constraining force $\mathbf{F}_{\text {constr. }}$ is applied to the $\mathrm{Ce}$ and $\mathrm{Sm}$ atoms ${ }^{25}$.

It is possible to show $^{27}$ that the VS thermostat is an approximate version of the Gaussian isokinetic thermostat ${ }^{23}$. We therefore use a term $\tilde{\alpha} \mathbf{p}_{i}$, where $\tilde{\alpha}$ is the thermostatting multiplier controlling the temperature in the Gaussian thermostat, in the EOM to symbolically represent the application of the VS thermostat.

The final dynamics of our simulations with the VS thermostat are thus represented by the following EOM:

$$
\begin{aligned}
& \dot{\mathbf{q}}_{i}=\frac{\mathbf{p}_{i}}{m_{i}}, \\
& \dot{\mathbf{p}}_{i}^{O}=\mathbf{F}_{i}^{D F T}+\mathbf{F}_{e}-\frac{m_{i}}{M} \mathbf{F}_{\text {tot }}, \\
& \dot{\mathbf{p}}_{i}^{C e, S m}=\mathbf{F}_{i}^{D F T}-\frac{m_{i}}{M} \mathbf{F}_{\text {tot }}+\mathbf{F}_{\text {constr. }}-\tilde{\alpha} \mathbf{p}_{i}^{C e, S m},
\end{aligned}
$$

During the NEMD simulation the quantity

$$
J(t)=\frac{1}{V} \sum_{i=1}^{N} c_{i} v_{i}(t),
$$

known as the color flux, is monitored. Here, $v_{i}(t)$ is the velocity component of atom $i$ in the direction of the field and $V$ is the volume of the simulation cell. Using the Green-Kubo relation for diffusion and linear response theory $^{23}$ the diffusivity, $D$, can be written:

$$
D=\frac{k_{B} T}{\rho_{c}} \lim _{t \rightarrow \infty} \lim _{F_{e} \rightarrow 0} \frac{\langle J(t)\rangle}{\left|\mathbf{F}_{e}\right|},
$$

where $k_{B}$ is the Boltzmann constant, $\rho_{c}$ is the density of color-charged atoms, i.e. atoms with non-zero color charge, and the angular brackets $\langle\ldots\rangle$ represent time averaging.

Our goal is thus to perform NEMD simulations for sufficient time as to reach convergence of the average color flux, i.e., $\langle J(t)\rangle=$ const, and then take its "zero field", $F_{e} \rightarrow 0$, limit.

The diffusivity can be related to the ionic conductivity by the Nernst-Einstein relation:

$$
\sigma=\frac{(Z e)^{2} \rho_{c}}{k_{B} T} D
$$

where $Z e$ is the charge of the charge carrier. In our case $Z e$ equals -2 elementary charges, i.e., the charge of an oxide ion. 


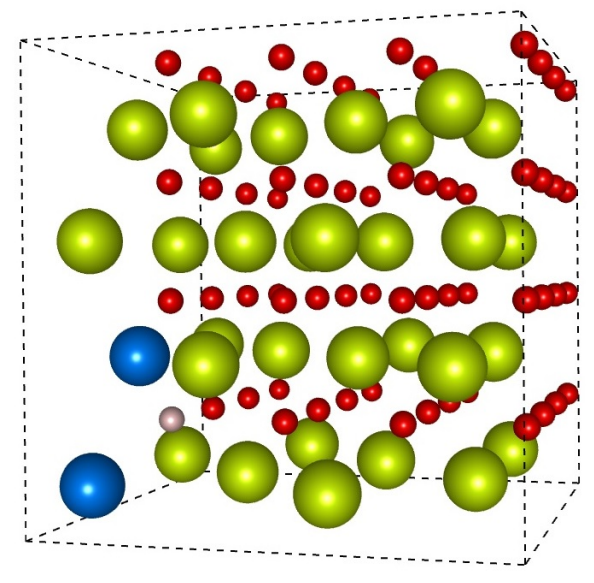

FIG. 1. The 95 atom, $6.25 \%$ Sm doped ceria, simulation cell used in this work. Green, blue and red spheres represent Ce, $\mathrm{Sm}$ and $\mathrm{O}$ atoms while the gray sphere is the vacancy.

If there are no diffusive jumps, i.e., all atoms simply oscillate thermally around their equilibrium positions, $\langle J\rangle$ must be 0 . In order to obtain a non-zero value for $\langle J\rangle$, diffusive "jumps", whereby oxygen atoms change their equilibrium positions, need to occur.

\section{COMPUTATIONAL DETAILS}

All calculations were performed using the Vienna $A b$ Initio Simulation Package (VASP) ${ }^{28-31}$, which had to be modified to support the color-diffusion algorithm. We used the generalized gradient approximation (GGA) in the form according to Perdew-Burke-Ernzerhof ${ }^{32}$ (PBE) to treat exchange and correlation effects. The plane-wave cutoff energy was set to $400 \mathrm{eV}$, and the Brillouin zone was sampled at the $\Gamma$ point. The projector augmented wave $^{33}$ (PAW) method was used to treat valence electrons. A special 3+ PAW potential was used for the Sm atoms where 5 of their $64 \mathrm{f}$ electrons were kept frozen in the core to avoid issues with the poor description of the localized character of the $4 \mathrm{f}$ electrons in standard DFT. A time step, $\Delta t=2 \mathrm{fs}$, was used to integrate the EOM using the Verlet algorithm. These parameters are suitable for the present system ${ }^{25}$.

The initial velocities of the atoms were drawn from a Maxwell-Boltzmann distribution at the appropriate temperature. After applying the external force, a 1 ps transient period was disregarded before gathering data. Calculations with structures equilibrated without an external field for up to 10 ps yielded no noticeable difference in the values of conductivity compared to those started from random velocities, and resulting data will thus be given from both types of calculations. We found that $\sim$ 40-70 ps of simulation time was enough to obtain proper convergence of the conductivity, depending on the temperature and the external field strength.

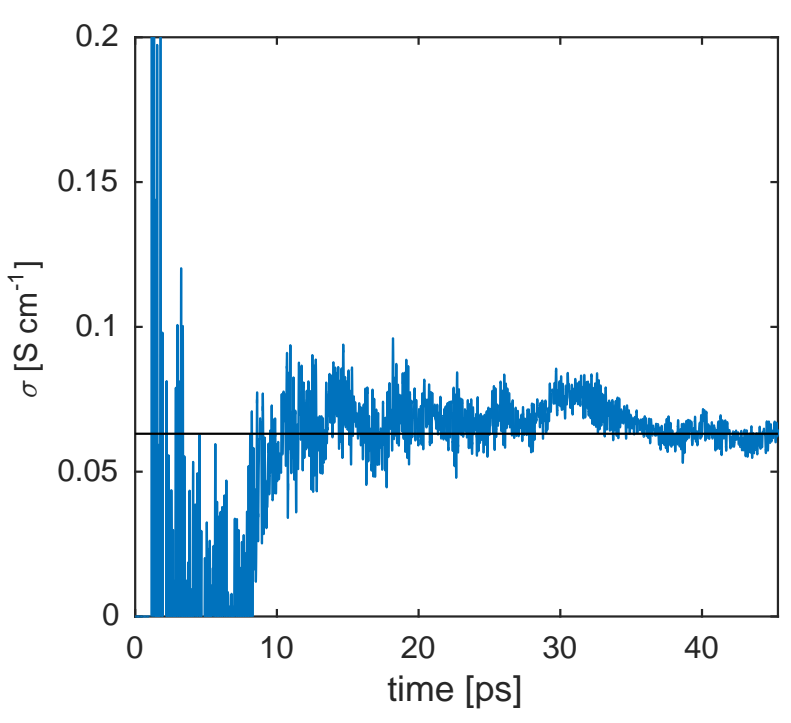

FIG. 2. Ionic conductivity, $\sigma$, as a function of simulation time. $\sigma$ appears converged after $\sim 40$ ps of simulation time.

The simulated supercell is a $2 \times 2 \times 2$ repetition of the $\mathrm{CeO}_{2}$ fluorite cubic unit cell with $2 \mathrm{Ce}$ atoms replaced by $\mathrm{Sm}$ atoms, corresponding to a dopant concentration of $6.25 \%$, and one $\mathrm{O}$ atom removed, leaving a vacancy in its place. This adds up to $30 \mathrm{Ce}, 2 \mathrm{Sm}$ and $63 \mathrm{O}$ atoms, i.e. a total of 95 atoms in the simulation cell (see Fig. 1). The lattice parameter was fixed at $5.42 \AA$, corresponding to the experimental value at the appropriate dopant concentration $^{3}$.

We choose a dopant configuration with the two Sm atoms as nearest neighbors (see Fig. 1). Since such a configuration with the oxygen vacancy in the first coordination shell to both of the $\mathrm{Sm}$ atoms will be the lowest energy state of the system, a trapping effect on oxygen vacancies is expected ${ }^{9,18}$. Accordingly, this configuration is convenient to use to investigate how well the present method reproduces the site preferences of oxygen vacancies in relation to the Sm dopants (see Sec. IV D 2). For comparison, we also perform two simulations with the dopants maximally separated in the simulation cell.

\section{RESULTS AND DISCUSSION}

\section{A. Calculating the steady state color flux}

To calculate the ionic conductivity (Eq. (5)), one has to evaluate the steady state color flux (SSCF), i.e. the $t \rightarrow$ $\infty$ limit of $\langle J(t)\rangle$. This can be done by simply calculating $\langle J(t)\rangle$ at every time step and observing the convergence of the ionic conductivity; an example of this is shown in Fig. 2 where the conductivity at an initial temperature of $T=1000 \mathrm{~K}$, as calculated by Eqs. (4) and (5), is shown as a function of simulation time. Convergence can be observed after $\sim 40 \mathrm{ps}$ of simulation time. The 


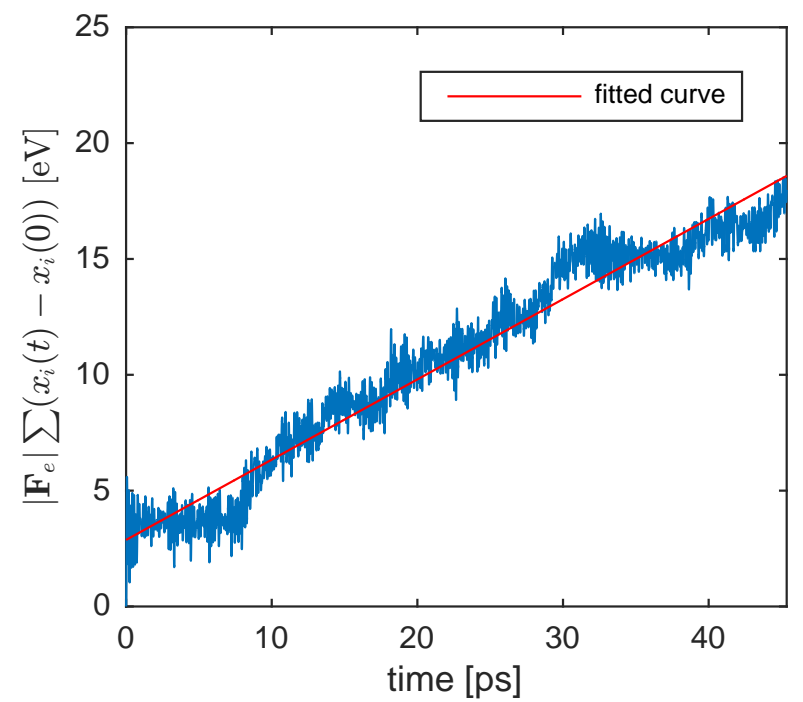

FIG. 3. Linear fit to identify the steady state color flux. The blue curve is the bracketed term on the right hand side of Eq. (6) and the red curve is its least squares linear fit. $\langle J\rangle$ is found from the slope of this linear fit using Eq. (7).

SSCF can, however, also be calculated by noting that $J(t)$, using Eq. (3), can be expressed as

$$
V\left|\mathbf{F}_{e}\right| J(t)=\frac{d}{d t}\left[\left|\mathbf{F}_{e}\right| \sum_{i=1}^{N}\left(x_{i}(t)-x_{i}(0)\right)\right],
$$

where $x_{i}$ is the projection of the position vector of atom $i$ on the direction of $\mathbf{F}_{e}$. Since we are expecting $\langle J\rangle=\langle J(t)\rangle=$ const it makes sense to fit the bracketed expression in Eq. (6) to a linear function $C_{1} t+C_{2}$, where $C_{1}$ and $C_{2}$ are constants. One can then find $\langle J\rangle$ from

$$
\langle J\rangle=\frac{C_{1}}{V\left|\mathbf{F}_{e}\right|} .
$$

Fig. 3 shows a plot of the bracketed expression on the right hand side of Eq. (6) along with its least squares linear fit. The slope of this linear fit is $C_{1}$, from which $\langle J\rangle$ is calculated using Eq. (7).

The two procedures just described give very similar values of the ionic conductivity. We choose to use the linear fit procedure in the following as it seems to be less sensitive to statistical fluctuations.

\section{B. The linear regime}

Eq. (4) implies that we must design our simulations such that we are able to evaluate the zero field limit of $\langle J(t)\rangle /\left|\mathbf{F}_{e}\right|$. If $\langle J(t)\rangle$ increases linearly with $\left|\mathbf{F}_{e}\right|$, the limit is trivial. The interval of external field strengths where such a linear dependence is observed is termed the linear regime.
Our previous study on Gd-doped ceria ${ }^{25}$ found a linear regime extending to $\left|\mathbf{F}_{e}\right| \approx 0.5 \mathrm{eV} / \AA$ at $T=1000$ $\mathrm{K}$, and it is reasonable to assume a similar extent in the present case. With this in mind, we performed calculations with $\left|\mathbf{F}_{e}\right|=0.4,0.5$, and $0.6 \mathrm{eV} / \AA$ at $T=1000$ K. The resulting $\langle J\rangle$ is shown in Fig. 4 (black line); here we have also used the fact that $\left|\mathbf{F}_{e}\right|=0 \Longrightarrow\langle J\rangle=0$. Linear behaviour can be observed up to $0.5 \mathrm{eV} / \AA$, while clear non-linearity is found for higher fields. Note that we have only performed calculations around the field values where we expect the linear regime to end. This allowed us to avoid time-consuming calculations at lower fields, where diffusive events are still rare and very long simulation times are needed for proper convergence.

The extent of the linear regime is related to the maximum field which does not change the physical mechanism of diffusion.

With this in mind, one expects the extent of the linear regime to be larger for systems where the atoms are performing smaller thermal oscillations and vice versa, i.e. we expect the linear regime to be temperature dependent.

To investigate this temperature dependence we performed tests, in the fashion described above, for temperatures of 800 and $1200 \mathrm{~K}$. These results are shown, along with those at $1000 \mathrm{~K}$, in Fig. 4. We note that the 0.5 $\mathrm{eV} / \AA$ field used at $1000 \mathrm{~K}$ is clearly outside the linear regime at $1200 \mathrm{~K}$.

The $0.6 \mathrm{eV} / \AA$ field appears to be slightly out of the linear regime at $800 \mathrm{~K}$. While it is quite likely that the edge of the linear regime at $800 \mathrm{~K}$ lies closer to $0.6 \mathrm{eV} / \AA$ than $0.5 \mathrm{eV} / \AA$, it is difficult to pinpoint it to higher accuracy than $\sim 0.1 \mathrm{eV} / \AA$. To be on the safe side, we therefore use the field of $0.5 \mathrm{eV} / \AA$, resulting in somewhat longer simulation times needed to gather proper statistics at $800 \mathrm{~K}$ than at higher temperatures.

To summarize, the optimized field strengths which could be safely used were $0.4,0.5$ and $0.5 \mathrm{eV} / \AA$ at temperatures 1200,1000 and $800 \mathrm{~K}$, respectively. At $900 \mathrm{~K}$ a field of $0.5 \mathrm{eV} / \AA$ was used, which from the above considerations is known to be safely inside the linear regime.

\section{Ionic Conductivity}

We used Eq. (5) to calculate the ionic conductivity, $\sigma$, at initial temperatures of 800, 900, 1000 and $1200 \mathrm{~K}$. As mentioned above, since the VS thermostat is only applied to the Ce/Sm subsystem, the actual average temperature of the simulations slightly differs from the initial temperature.

Four simulations were performed at an initial temperature of $1000 \mathrm{~K}$, where the initial vacancy position was chosen differently in each case. Values of $\sigma$ and average temperature are given in Table I, where values from experiments and $\mathrm{KMC}$ and classical MD simulations are provided for comparison.

The moderate difference in conductivity values from 


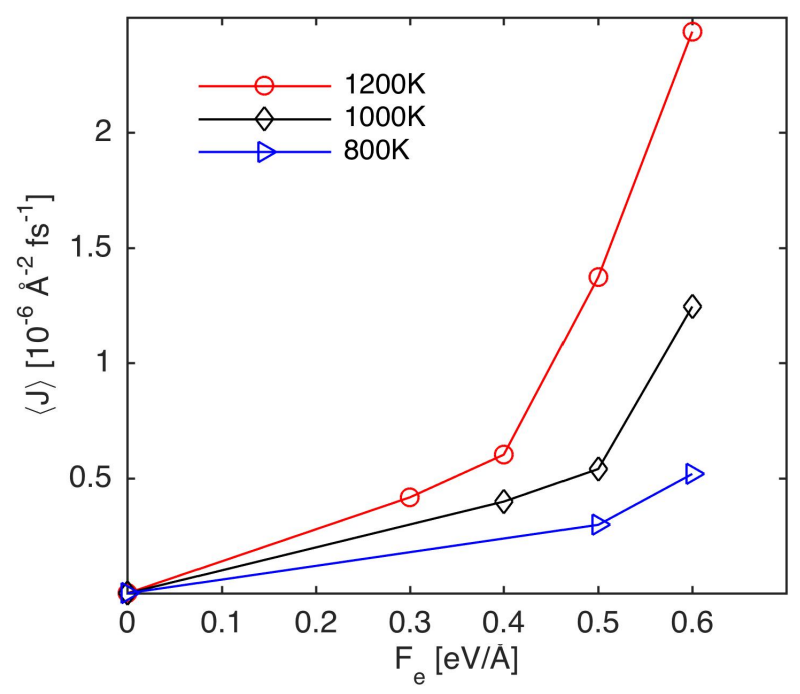

FIG. 4. Identification of the linear regime for different temperatures. Linear relations between the flux and the field is observed up to field strengths of $0.5 \mathrm{eV} / \AA, 0.5 \mathrm{eV} / \AA, 0.4$ $\mathrm{eV} / \AA$ for temperatures 800,1000 and $1200 \mathrm{~K}$, respectively.

\begin{tabular}{lcccc}
\hline \hline \multicolumn{4}{c}{ Ionic conductivity, $\sigma$, at $\mathbf{T} \sim \mathbf{1 0 4 5 - 1 0 7 5 ~ K}$} \\
\hline Method & $\mathrm{T}(\mathrm{K})$ & $\% \mathrm{Sm}$ & $\sigma\left(\mathrm{Scm}^{-1}\right)$ & Source \\
\hline NEMD & 1049 & 6.25 & $7.4 \cdot 10^{-2}$ & This work \\
& 1062 & & $7.9 \cdot 10^{-2}$ & \\
& 1046 & & $8.8 \cdot 10^{-2}$ & \\
& 1053 & & $6.9 \cdot 10^{-2}$ & \\
\hline KMC & 1073 & 5 & $0.52 \cdot 10^{-2}$ & Dholabhai et al. \\
& 1073 & 10 & $0.83 \cdot 10^{-2}$ & \\
\hline CMD & 1073 & 5 & $2.5 \cdot 10^{-2}$ & Burbano et al. \\
& 1073 & 10 & $2.8 \cdot 10^{-2}$ & \\
\hline Experiment & 1073 & 5 & $5.0 \cdot 10^{-2}$ & Zha et al. ${ }^{3}$ \\
& 1073 & 10 & $6.9 \cdot 10^{-2}$ & \\
\hline \hline
\end{tabular}

TABLE I. Ionic conductivities and mean temperatures for simulations started at $T 1000 \mathrm{~K}$, obtained in this work. Results from classical $\mathrm{MD}^{7}, \mathrm{KMC}^{8}$ and experiment ${ }^{3}$ are also given.

the four simulations is attributed to the different paths on which the vacancy travels through the simulation cell. The time the vacancy spends in a certain position is related to the proximity of the Sm atoms; this is discussed in more detail in section IV D 2. A larger fraction of all vacancy paths in the simulation cell is covered, at the same computational expense, by initiating different simulations with the vacancy in different positions, compared to performing a single, very long, simulation. Averages of the conductivities obtained from the different simulations can then be taken.

At each of the three remaining temperatures three additional simulations were performed.

Results from all aforementioned simulations are shown in Fig. 5, where for each temperature we display the average conductivity value for different simulations started at the same thermostat temperature. These conductivities are placed at the average temperature for the different simulations started at a specific thermostat temperature. The supplied error bars are standard deviations.

It is clear that our results slightly overestimate the ionic conductivity, as compared to experiment. This is to be expected since we treat an ideal bulk while experimental values are given as total conductivities, which have contributions from both grains and grain boundaries ${ }^{3,5}$. Other features not taken into account include different distributions of dopants, thermal expansion and effects from the limited size of the simulation cell, among others. We note, however, that the agreement with experiment is still very good and, in particular, of a similar or better degree than previous theoretical efforts.

For temperatures up to $\sim 1000 \mathrm{~K} \sigma$ roughly follows the Arrhenius behavior (see Fig. 5) with an activation energy of $0.41 \mathrm{eV}$. Experimental ${ }^{3}$ activation energies extracted for the same temperature range are $0.65 \mathrm{eV}$ and $0.79 \mathrm{eV}$ for 10 and 5 at. \% dopant concentration, respectively. This underestimation is consistent with the fact that we have made no attempt to include effects from thermal expansion. The thermal expansion of the lattice would likely further increase the conductivity increment when temperature is raised, resulting in a larger activation energy within the Arrhenius model.

At higher temperatures the conductivity deviates from a straight line. Certainly, the anharmonic lattice vibrations are among the underlying reasons for this deviation but a deeper analysis is beyond the scope of this work. We note that there is no reason in theory to expect the conductivity to obey the Arrhenius equation at high temperature and the experimental grain conductivity in Sm-doped ceria ${ }^{4}$ clearly demonstrates the corresponding deviation.

\section{Microscopic details of the oxygen diffusion}

\section{Diffusion mechanism}

While the agreement of the calculated ionic conductivity with experimental values gives a certain amount of confidence in the present method, Eq. (5) does not give any insight into the mechanism by which the diffusion takes place during our simulations. It is, however, as previously noted, well known that oxygen ions diffuse via jumps into neighboring vacant lattice sites along the $\langle 100\rangle$ directions. Since our simulations are performed with a perturbing field along the [111] direction, there is no guarantee that the diffusion process is still the correct one, i.e., we need to make sure that the field does not bias the diffusion process to happen in a different, unphysical, way. Fig. 6 shows projections of the oxygen atom trajectories onto the (001) and (010) planes of the simulation cell for a single simulation with initial temperature $T=1000 \mathrm{~K}$. The trajectories are generated from the positions of the oxygen atoms every 30 fs for a total time of 


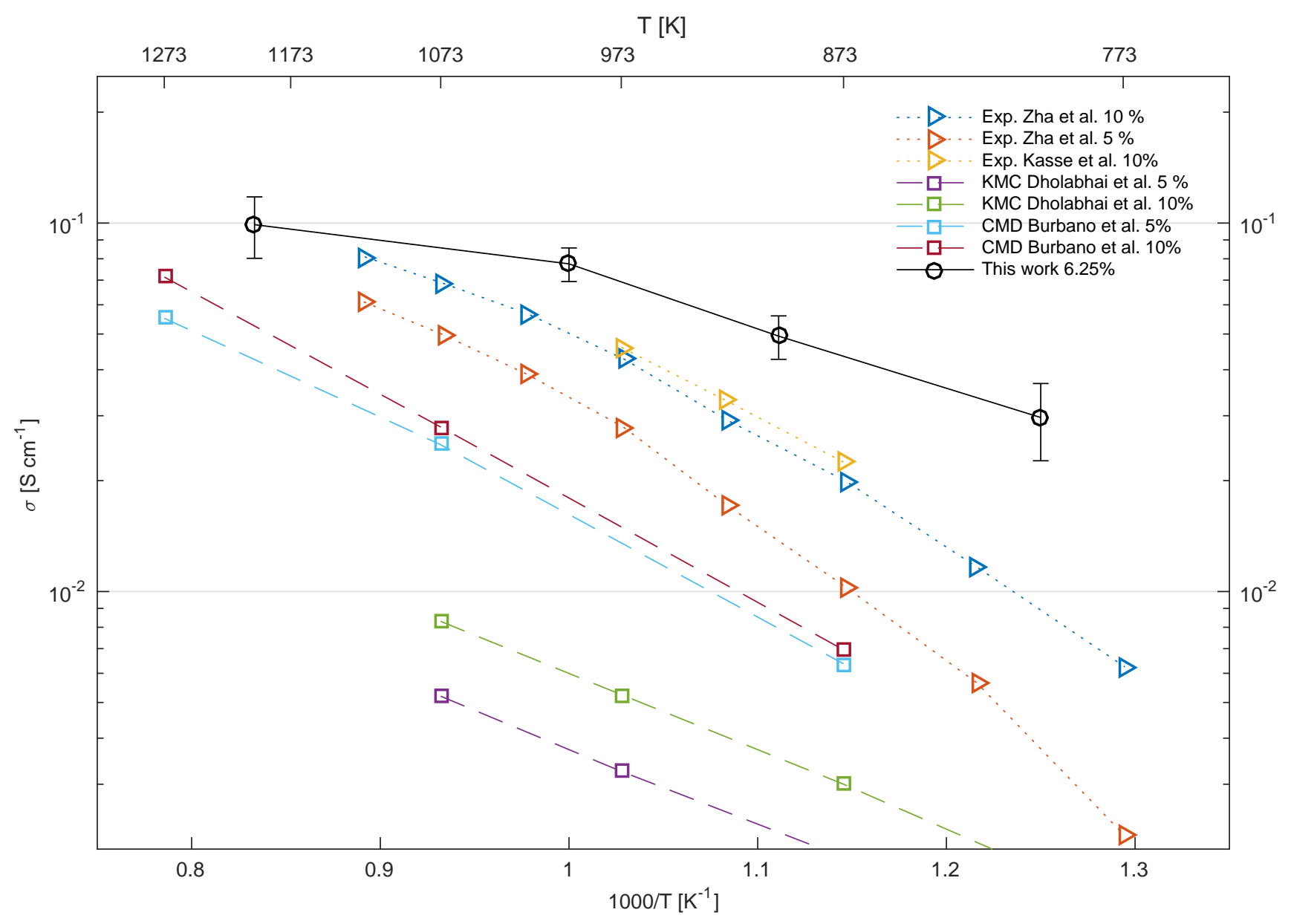

FIG. 5. Ionic conductivities, $\sigma$, plotted versus inverse temperature. The solid black line corresponds to the results from this work, error-bars are standard deviations. Theoretical results from classical $\mathrm{MD}^{7}$ and $\mathrm{KMC}^{8}$ simulations are given by the dashed lines. Dotted lines present experimental results $^{3,5}$.

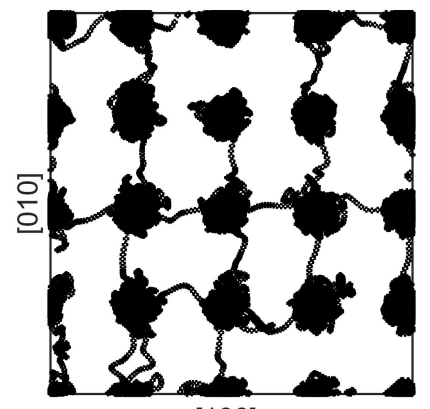

[100]

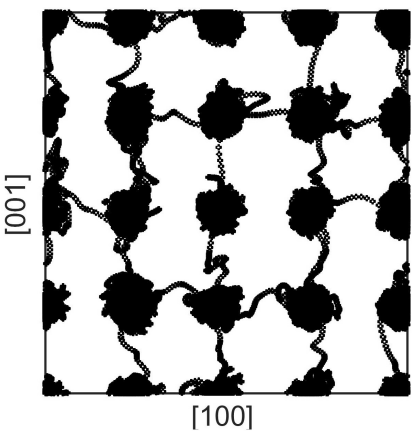

[100]
FIG. 6. Projections of the trajectories of the oxygen atoms onto the (001) and (010) planes, from which it can be seen that diffusion occurs by migration along the $\langle 100\rangle$ directions.

58 ps. The black blobs are centered around the equilibrium lattice positions and one can see that the movement between these occurs only parallel to the coordinate axes, which clearly indicates that the diffusion process occurs via oxygen jumps along the $\langle 100\rangle$ directions.

\section{Influence of dopants on oxygen vacancy migration}

Another feature of the diffusion process in doped ceria is the influence of the dopant atoms. Previous $T=0 \mathrm{~K}$ DFT calculations have shown that rare earth dopants, such as Sm, strongly influence the oxygen diffusion. In particular, it is found that oxygen vacancies prefer positions closer to $\mathrm{Sm}$ atoms ${ }^{9,18}$. We thus need to make sure that the influence of the external field does not change this site preference implied by the Sm dopants.

To investigate the effect of the Sm atoms on the diffusion process, the position of the vacancy was identified (as the ideal lattice position where an $\mathrm{O}$ atom is missing) every 10 fs during the four combined runs $(\sim 220 \mathrm{ps}$ in total) at $1000 \mathrm{~K}$, and its position in relation to the two $\mathrm{Sm}$ atoms was recorded. Fig. 7 shows the vacancy-Sm partial 


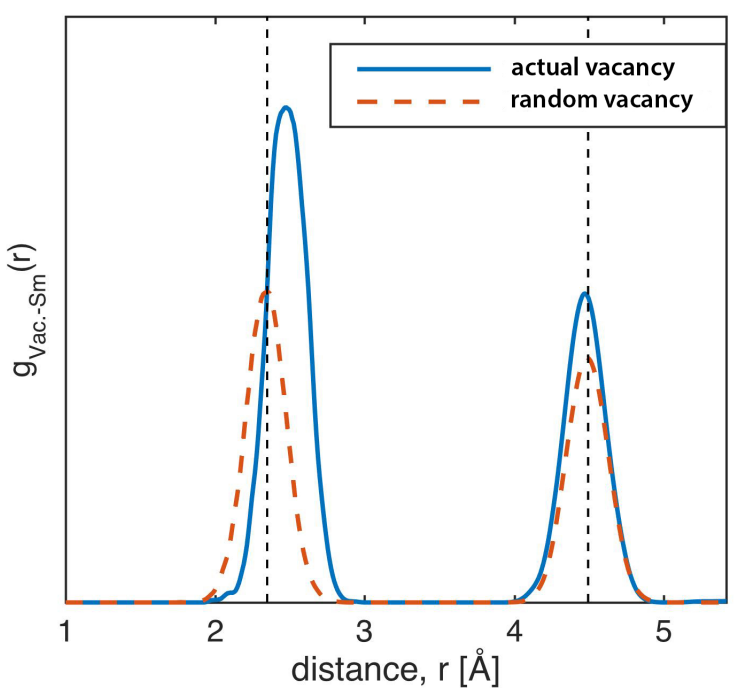

FIG. 7. Partial radial distribution function, $g_{V a c .-S m}(r)$, obtained from atomic positions every $10 \mathrm{fs}$ for $\sim 220 \mathrm{ps}$ of simulation time at $1000 \mathrm{~K}$. The solid blue curve is generated from the actual Sm and vacancy positions while the dashed red curve is obtained from random vacancy positions. A clear site preference of the vacancy close to the dopants is observed.

radial distribution function $(\mathrm{RDF}), g_{V a c .-S m}(r)$, generated from the gathered data, for values of $r$ up to half the length of the simulation box edge. The blue solid curve is generated from the actual vacancy positions while the red dashed curve is generated from randomly placing the vacancy on a position of the $\mathrm{O}$ sublattice; in this case the $\mathrm{Sm}$ atoms have no influence on the vacancy positions. The difference between these two curves reveals the influence of the Sm dopants on the site preference of the vacancy. The average distances obtained from the atoms sitting in their ideal lattice positions are indicated as dotted black vertical lines where the two peaks correspond to vacancies in the first and second coordination shell of an Sm atom, respectively

The area under the peaks corresponding to the first and second coordination shells is greater for the actual vacancy positions observed in the simulation compared with the random vacancy distribution. Therefore, the vacancy prefers to sit in close proximity to the Sm atoms. This is in agreement with the conclusions of the previously mentioned static DFT studies ${ }^{9,18}$.

We also observe a shift of the first peak of $\sim 0.16$ $\AA$ which indicates that the Sm dopants have shifted their equilibrium positions away from the vacancy, which is also in agreement with previous zero Kelvin DFT calculations ${ }^{9}$.

A subtle issue concerning the long time spent by the vacancy in the first coordination shell of both Sm atoms in the simulation cell, denoted as $(1,1)$ positions, should be noted. Since we only use color charges $c_{i}=1$ the [111] external field direction only allows for three out of

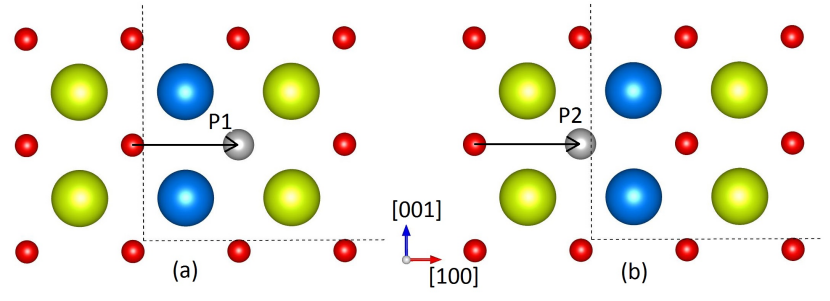

FIG. 8. The two vacancy (gray circle) positions, a) and b), in the simulation cell that are nearest neighbor to both Sm (blue circle) dopants. Red and green circles are O and Ce atoms, respectively, and the dotted black line marks the edge of the simulation cell (see Fig. 1). The path P1 has a migration barrier twice as high as P2, which makes the two positions non-equivalent and the vacancy will thus spend more time in position (a) than (b).

the normally six possible diffusion paths into a certain vacancy position. Fig. 8 shows a $2 \mathrm{D}$ view of the two existing $(1,1)$ positions in the simulation cell (Fig. 8 (a) and (b); see also Fig. 1). These two positions are non-equivalent since the paths labeled P1 and P2 differ in migration barriers. Since P1 passes between two Sm dopants its migration barrier is twice as high $\mathrm{P} 2^{9}$. This will result in an additional trapping effect for the vacancy in position (a) as compared to (b) which is unphysical since it emerges only from the distribution of the color charges and external field. The influence of this artificial non-equivalence of $(1,1)$ positions will, however, only have a minor effect on the RDF in Fig. 7 since combination of the two $(1,1)$ positions allows for all possible non-equivalent diffusion paths into this particular type of vacancy position.

To make sure that this effect has no large influence on the ionic conductivity, two additional simulations were performed at $1000 \mathrm{~K}$ with the Sm dopants maximally separated in the simulation cell. The resulting conductivities were 0.079 and $0.077 \mathrm{Scm}^{-1}$, very similar to those obtained with nearest neighbor dopants.

\section{CONCLUSION}

To conclude, a first-principles non-equilibrium molecular dynamics study on the diffusion process and ionic conductivity in bulk Sm-doped ceria has been performed using the color-diffusion algorithm. We have shown that the strongest external field that can be used while keeping the system in the domain of linear response is temperature dependent. Results on the ionic conductivity in good agreement with experimental data have been presented, and we have also presented evidence that the microscopic details of the diffusion process are well reproduced by the method; a diffusion mechanism consisting of oxygen ion jumps in the $\langle 100\rangle$ directions has been demonstrated and the influence of the Sm dopants on the site preference of the vacancy has also been shown to match well the known trends. 


\section{ACKNOWLEDGMENTS}

We acknowledge Dr. O. Hellman for valuable suggestions and discussions. N.V.S., O.Y.V., and J.O.N. acknowledge the financial support of Swedish Energy Agency (STEM) (Project No. 35515-1), Carl Tryggers Foundation (Project No. CTS 14:433), and the Swedish Research Council (VR) (Project No. 2014-5993). The support from Swedish Research Council (VR) (Project
No. 2014-4750), LiLi-NFM, and the Swedish Government Strategic Research Area Grant in Materials Science to the AFM research environment at LiU are acknowledged by S.I.S and J.K. The computations were performed on resources provided by the Swedish National Infrastructure for Computing (SNIC) at the PDC Centre for High Performance Computing (PDC-HPC) and the National Supercomputer Center (NSC).
* johan.klarbring@liu.se

1 A. Trovarelli, Catalysis by ceria and related materials, Vol. 2 (World Scientific, 2002)

2 D. J. L. Brett, A. Atkinson, N. P. Brandon, and S. J. Skinner, Chemical Society Reviews 37, 1568 (2008).

3 S. Zha, C. Xia, and G. Meng, Journal of Power Sources 115, 44 (2003).

4 S. Kuharuangrong, Journal of Power Sources 171, 506 (2007)

5 R. M. Kasse and J. C. Nino, Journal of Alloys and Compounds 575, 399 (2013).

6 W. Shen, J. Jiang, and J. L. Hertz, The Journal of Physical Chemistry C 118, 22904 (2014).

7 M. Burbano, S. Nadin, D. Marrocchelli, M. Salanne, and G. W. Watson, Phys. Chem. Chem. Phys. 16, 8320 (2014).

8 P. P. Dholabhai and J. B. Adams, Journal of Materials Science 47, 7530 (2012).

9 D. A. Andersson, S. I. Simak, N. V. Skorodumova, I. A. Abrikosov, and B. Johansson, Proceedings of the National Academy of Sciences of the United States of America 103, 3518 (2006).

10 B. O. H. Grope, T. Zacherle, M. Nakayama, and M. Martin, Solid State Ionics 225, 476 (2012).

11 A. Gotte, D. Spångberg, K. Hermansson, and M. Baudin, Solid State Ionics 178, 1421 (2007).

12 C. Huang, W. Wei, C. Chen, and J. Chen, Journal of the European Ceramic Society 31, 3159 (2011).

13 Z. Cui, Y. Sun, and J. Qu, Solid State Ionics 226, 24 (2012).

14 M. Nakayama and M. Martin, Physical Chemistry Chemical Physics : PCCP 11, 3010 (2009).

15 a. Tarancón, a. Morata, F. Peiró, and G. Dezanneau, Fuel Cells 11, 26 (2011).

16 S. Grieshammer, B. O. H. Grope, J. Koettgen, and M. Martin, Physical Chemistry Chemical Physics 16, 9974
(2014).

17 S. Vives and C. Meunier, Solid State Ionics 283, 137 (2015).

18 A. Ismail, J. Hooper, J. B. Giorgi, and T. K. Woo, Physical Chemistry Chemical Physics : PCCP 13, 6116 (2011).

19 M. Rushton and A. Chroneos, Scientific Reports 4 (2014).

${ }^{20}$ H. Wang, A. Chroneos, and U. Schwingenschlögl, The Journal of Chemical Physics 138, 224705 (2013).

21 A. R. Genreith-Schriever, P. Hebbeker, J. Hinterberg, T. Zacherle, and R. A. De Souza, The Journal of Physical Chemistry C 119, 28269 (2015).

22 M. Alaydrus, M. Sakaue, S. M. Aspera, T. D. Wungu, N. H. Linh, T. P. Linh, H. Kasai, T. Ishihara, and T. Mohri, Journal of the Physical Society of Japan 83, 094707 (2014).

23 D. J. Evans and G. Morriss, Statistical Mechanics of Nonequilibirum Liquids, 2nd edition. (Cambridge University Press, 2008).

24 P. C. Aeberhard, S. R. Williams, D. J. Evans, K. Refson, and W. I. F. David, Physical Review Letters 108, 1 (2012).

25 J. O. Nilsson, O. Y. Vekilova, O. Hellman, J. Klarbring, S. I. Simak, and N. V. Skorodumova, Phys. Rev. B 93, 024102 (2016).

26 This is opposed to the case where the momenta of all the atoms are scaled: $\sum \mathbf{p}_{i}=\mathbf{0} \Longrightarrow S \sum \mathbf{p}_{i}=\mathbf{0}$, where $\mathrm{S}$ is the scaling factor.

27 S. Nose, Prog. Theor. Phys. Suppl. , 46 (1991).

28 G. Kresse and J. Hafner, Physical Review B 48, 13115 (1993).

29 G. Kresse and J. Hafner, Physical Review B 49, 14251 (1994).

30 G. Kresse, Physical Review B 54, 11169 (1996).

31 G. Kresse and J. Furthmüller, Computational Materials Science 6, 15 (1996).

32 J. P. Perdew, K. Burke, and M. Ernzerhof, Physical Review Letters 77, 3865 (1996).

33 P. E. Blöchl, Physical Review B 50, 17953 (1994). 